\title{
Sexual Rehabilitation After Pelvic Radiotherapy and Vaginal Dilator Use Consensus Using the Delphi Method
}

\author{
Rinske Maria Bakker, MSc, * Moniek M. ter Kuile, PhD, *Willemijn M. Vermeer, PhD, * \\ Remi A. Nout, MD, PhD, † Jan Willem M. Mens, MD, $\neq$ Lena C. van Doorn, MD, PhD, $\S$ \\ Cor D. de Kroon, MD, PhD, * Willemien C.P. Hompus, BSc, $\S$ Cora Braat, MANP, \\ and Carien L. Creutzberg, MD, PhD †
}

\begin{abstract}
Objective: This study aimed to reach consensus among professional experts on patient information provision and support regarding sexual rehabilitation and vaginal dilator use after radiotherapy (RT) for gynecological cancers.

Methods: A 3-round online Delphi study was conducted among 10 radiation oncologists, 10 gynecologic oncologists, and 10 oncology nurses from 12 gynecological cancer centers providing RT for gynecological tumors. We assessed the desired content and provider of instructions and patient support regarding sexuality and vaginal dilator use. Responses were measured on a 7-point scale varying from "totally disagree" to "totally agree." Consensus was reached when $70 \%$ of participants' answers fell within 2 scale categories with an interquartile range less than or equal to 1 .

Results: The panel agreed that information about sexual rehabilitation using vaginal dilators should be provided by radiation oncologists before treatment. Information should always be provided to sexually active cervical and vaginal cancer patients younger than 70 years. Tailored information was recommended for vulvar and endometrial cancer patients, patients older than 70 years, and sexually inactive patients. Preferably, specifically trained oncology nurses should give psychological and practical support. Participants recommended vaginal dilation to prevent vaginal adhesions, tightening, and shortening. The panel advised to start dilation around 4 weeks after treatment to perform dilation 2 to 3 times a week for 1 to 3 minutes and to continue dilation for 9 to 12 months. Plastic dilator sets were considered the most suitable type of dilator.
\end{abstract}

Conclusions: Consensus was reached on patient information provision and support during sexual rehabilitation after RT for gynecological cancers. Results were used to develop a sexual rehabilitation intervention.

Key Words: Gynecological cancer, Pelvic radiotherapy, Sexual rehabilitation, Vaginal dilator use, Delphi method

Received May 7, 2014, and in revised form August 3, 2014.

Accepted for publication August 3, 2014.

(Int J Gynecol Cancer 2014;24: 1499-1506)

Departments of *Gynecology, and †Clinical Oncology, Leiden University Medical Center, Leiden; and Departments of $\$$ Radiotherapy, and §Gynecology, Erasmus Medical Center Cancer Institute, Rotterdam, the Netherlands.

Address correspondence and reprint requests to Rinske Maria Bakker,

MSc, Department of Gynecology, Leiden University Medical

Copyright (C) 2014 by IGCS and ESGO

ISSN: $1048-891 \mathrm{X}$

DOI: $10.1097 / \mathrm{IGC} .0000000000000253$
Center, Zone VRSP, PO Box 9600, 2300 RC Leiden, the Netherlands. E-mail: R.M.Bakker@lumc.nl.

This study was funded by the Dutch Cancer Society and Alpe d'HuZes foundation (UL2011-5245).

Supplemental digital content is available for this article. Direct URL citation appears in the printed text and is provided in the HTML and PDF versions of this article on the journal's Web site (www.ijgc.net).

The authors declare no conflicts of interest. 
In the Netherlands, each year, 4500 women are diagnosed with gynecological cancer, ${ }^{1}$ among which 1900 are diagnosed with endometrial cancers, 750 are diagnosed with cervical cancers, and 60 are diagnosed with vaginal cancers. About $35 \%$ of these women are treated with pelvic radiotherapy (RT), either as primary or postsurgical treatment. ${ }^{2}$ Treatment for gynecological cancer may cause physical and psychological adverse effects that interfere with the women's sexuality. Reported sexual problems among gynecological cancer survivors are tightened and shortened vagina, diminished lubrication, dyspareunia, postcoital bleeding, and loss of sexual desire, enjoyment, and satisfaction. ${ }^{3-7}$ Most studies agreed that the negative effect of gynecological cancer treatment on sexual functioning was more pronounced when treatment included RT, compared with surgery alone. ${ }^{8-10}$ The negative effect of RT is attributed to decreased lubrication, shortening, and tightening of the vagina as a result of formation of fibrosis. ${ }^{10,11}$

Few studies investigated the effect of the regular use of vaginal dilators on the development or prevention of vaginal stenosis after treatment with RT. These studies showed that regular vaginal dilator use is associated with less vaginal shortening and/or tightening. ${ }^{12-14}$ It is not clear how the (changes in) vaginal dimensions were assessed in these studies nor what the cause-and-effect relationship was between dilator use and the vaginal measurements. Although more empirical evidence is needed, ${ }^{15}$ in clinical practice, regular dilator use is found to reduce the risk of shortening and/or tightening due to adhesions and fibrosis. Dilator use is therefore advocated in many guidelines and reviews. ${ }^{16-18}$

It is important to provide consistent and uniform evidence-based counseling regarding when and how dilators should be used. ${ }^{19-21}$ In 2 survey studies from the United Kingdom and Australia, ${ }^{22,23}$ there was consensus among professionals that women undergoing pelvic RT for gynecological malignancies should receive information about vaginal dilation. $^{22}$ In addition, professionals recommended to insert a vaginal dilator during 5 to 10 minutes. ${ }^{22,23}$ Other recommendations were inconsistent. ${ }^{22,23}$ No consensus existed regarding the most appropriate time interval after RT, the frequency and duration of dilator use, dilator sizes offered, insertion techniques, or the appropriateness of dilator use among sexually inactive patients. ${ }^{22,23}$ Moreover, there was no consensus on the content of instructions regarding patients' sexual rehabilitation.

There is a clear need for consensus on all of these aspects to further investigate the efficacy of a standardized procedure of sexual rehabilitation and dilator use after RT. Consensus is needed specifically on which specific gynecological cancer patients should receive information about sexual rehabilitation and dilator use; what type of health care provider should provide this information, counseling, and support; and what should be the practical guidelines for use of vaginal dilation.

The Delphi method proved to be an anonymous and economic tool to reach consensus on best practice issues in health care settings. ${ }^{24-28}$ At first, a questionnaire addressing the opinion of an expert panel is assessed. Then a second questionnaire is developed that is based on the first questionnaire without the statements on which consensus was reached. It is offered to the panel containing anonymous feedback on the panels' agreement. This encourages the panel to reconsider their first response to the statements.

This study used the Delphi method and aimed to determine clear recommendations on the content and procedures of patient information provision and support regarding sexual rehabilitation and vaginal dilator use after RT for gynecological cancers. This was done conducting a 3-round online Delphi study among recognized gynecologic oncology professionals from different cancer centers and with various areas of clinical expertise.

\section{MATERIALS AND METHODS}

\section{Participants}

Eligible participants were radiation oncologists, gynecologic oncologists, and oncology nurses, with recognized clinical expertise in the treatment of gynecological cancer patients receiving pelvic RT and expert knowledge on vaginal dilation in this population. It was expected that 30 participants, representing all Dutch gynecological cancer centers and each of the 3 specialisms equally, would create a heterogeneous and representative panel. ${ }^{25-28}$

Clinicians who participated in the Dutch gynecological cancer network received an invitation e-mail, together with a brochure explaining the study content and the Delphi method, and an online informed consent form. Nonresponders were approached by phone 1 week later. Before enrolment, it was ascertained that participants had the intention to complete all rounds of the study and had access to the Internet. At the conclusion of the study, the participants received a 20-euro ( US \$27) gift voucher as a token of appreciation. The study was approved by the Leiden University Medical Center Medical ethics committee.

\section{Questionnaire}

The questionnaire consisted of statements and questions addressing participants' opinions on 7 different categories (Table 1). The questionnaire was developed based on literature on sexuality and vaginal dilation after pelvic RT $^{19-23,29}$ and previous in-depth interviews with professionals involved in gynecological cancer treatment. Items were pilot-tested on comprehensibility among 5 experienced researchers in the field of gynecologic oncology and/or conducting Delphi studies. Answers were measured using a 7-point Likert scales varying from 1 (totally disagree) to 7 (totally agree) $(n=53)$, singlechoice $(n=8)$, and multiple-choice questions $(n=6)$. The questionnaire also consisted of items measuring demographic and work-related characteristics (eg, age and the years of experience in the field).

\section{The Delphi Process}

The present Delphi method was based on a frequently published standard design. ${ }^{24-28}$ The questionnaire elicited responses in 3 rounds. After each round, the degree of consensus was calculated. In case no consensus was reached on an item, the group response was fed back to the participants 
TABLE 1. Description of the 7-item categories used in the questionnaire

\begin{tabular}{ll}
\hline Item Category & \multicolumn{1}{c}{ Description of the Item Category } \\
\hline Responsibility & $\begin{array}{c}\text { Responsibilities that radiation oncologists, gynecological oncologists, and oncology nurses have } \\
\text { regarding their patients' sexual rehabilitation (eg, providing practical advice on how to cope } \\
\text { with sexual problems) } \\
\text { Specific patient groups that should receive information regarding sexual rehabilitation using } \\
\text { vaginal dilators } \\
\text { Type of dilator that is best advised }\end{array}$ \\
$\begin{array}{l}\text { Vaginal dilator } \\
\text { Rationale } \\
\text { Content instructions }\end{array}$ & $\begin{array}{l}\text { The rationale that health care providers should use to advise the use of dilators } \\
\text { Information and instructions that should be provided regarding the use of vaginal dilators and } \\
\text { sexual intercourse during sexual rehabilitation } \\
\text { Type of health care provider that should provide information regarding sexual rehabilitation and } \\
\text { Information provision } \\
\text { vaginal dilator use, the time interval at which information should be provided, and the } \\
\text { informational resource that should be used } \\
\text { Type of health care provider that should provide sexual health support during rehabilitation and } \\
\text { dilator use and to what extent sexul health support should be given by a radiation oncologist, } \\
\text { gynecological oncologist, or oncology nurse }\end{array}$ \\
\hline
\end{tabular}

in the next round. Participants were asked to comment on their answers in case it differed from the group response. Items on which no consensus was reached, and that, according to the comments by participants, seemed to be unclear were adapted.

The degree of consensus on the 6 multiple-choice questions that were used in round 1 could not be calculated. Therefore, the multiple-choice questions were not counted as part of the total number of items in round 1 and reformulated as 25 Likert scale statements in the next round.

An online Delphi study was conducted to allow anonymous inclusion of professionals across diverse centers and expertise and avoid that a specific expert might dominate the consensus process. Participants were asked to fill in each questionnaire within 2 weeks. Nonresponders were sent a reminder by e-mail and, if necessary, received a subsequent phone call after 1 week.

\section{Consensus}

It was decided beforehand that consensus was reached when at least $70 \%$ of participants' answers fell within the 2 lowest or highest answer categories on a Likert scale or within 1 category on a single-choice or multiple-choice question. In addition, to reach consensus, an interquartile range (IQR) of less than or equal to 1 was required. An IQR is a measure of statistical dispersion representing the distance between the 25th and the 75th percentile. A smaller IQR signifies a large consensus, and an IQR less than or equal to 1 represents good consensus on a 7-point Likert scale. ${ }^{27,28}$ When the degree to which consensus was reached differed between the 3 specialisms, this will be reported.

\section{Statistical Analyses}

Descriptive statistics (percentages and IQRs) were calculated to measure consensus. The group response on items on which no consensus was reached was fed back to the participants in round 2 by stating the median and in round 3 by stating the median together with the modus. All statistical analyses were done using the Statistical Package for the Social Sciences version 20.0.

\section{RESULTS}

\section{Participants}

Thirty-two clinicians were approached, of which 30 agreed to participate with a mean age of 48 years (see Fig. 1 for more information on the Delphi panel characteristics). Experts from 3 different disciplines and all 12 gynecological cancer centers in the Netherlands were included. Twentyseven participants had more than 5 years of experience within the field of gynecologic oncology $(90 \%)$, and 24 participants regularly or often gave patients sexological support $(80 \%)$. All participants $(100 \%)$ completed the 3 rounds.

\section{Results}

The specific results of the consensus rounds are shown in Supplemental Digital Contents 1 and 2, which illustrate all items that reached consensus (in round 1,2, and 3) and all items that did not reach consensus, respectively (available at http://links.lww.com/IGC/A230 and http://links.lww.com/ IGC/A231).

\section{Consensus Round 1}

Consensus was reached for 22 of 50 items in round 1 (44\%; Supplemental Digital Content 1, http://links.lww.com/ IGC/A230). After round 1, 8 items were formulated differently based on comments by participants. Furthermore, 1 item was removed from the questionnaire because this question was not specific enough.

The degree with which consensus was reached was equal between the 3 professional groups except for 1 item. The gynecologic oncologists reached the consensus that it is 


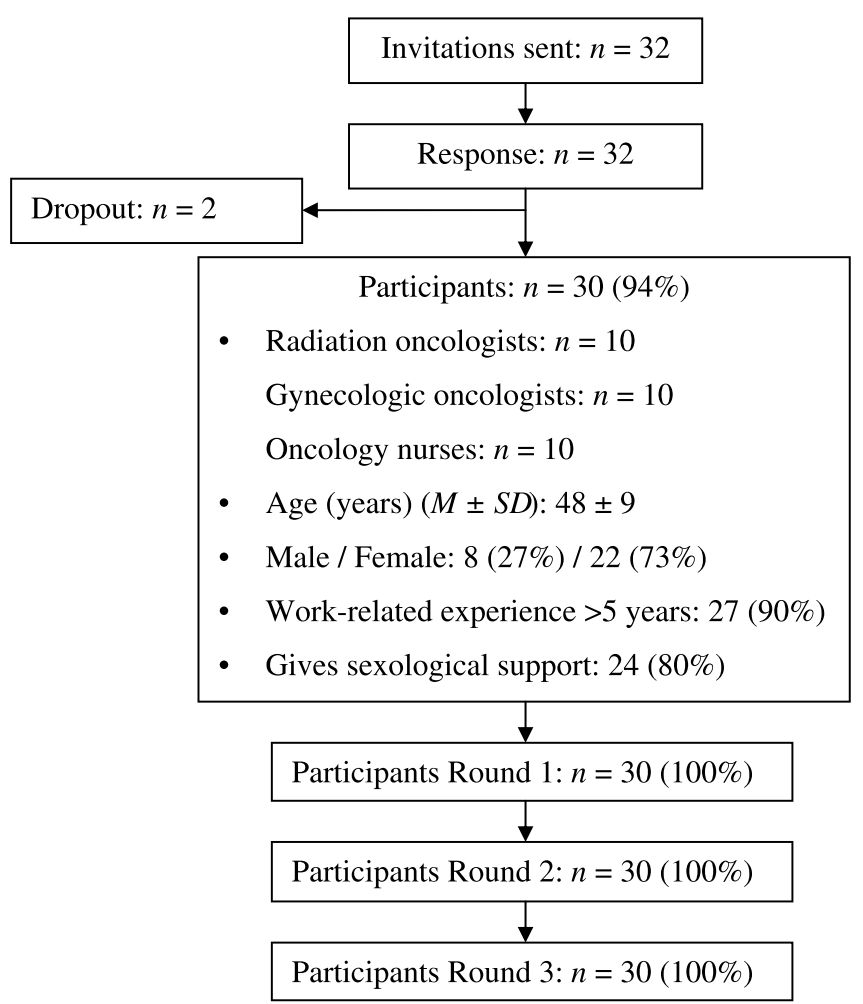

FIGURE 1. Delphi panel characteristics.

part of their responsibility to evaluate their patients' sexual functioning $(\mathrm{n}=10 ; 100 \%$ agreed; IQR, 1.00$)$. The radiation oncologists almost reached the same consensus $(n=8 ; 80 \%$ agreed; IQR, 1.25). However, the oncology nurses disagreed (IQR, 4.50) about whether this is their responsibility.

The panel did uniformly agree that it is important to give patients advice on how to cope with fear for sexual contact after treatment and, if necessary, to refer patients to a sexologist after treatment with RT for gynecological cancers. Furthermore, participants agreed that all sexually active cervical and vaginal cancer patients with a partner, younger than 70 years, should always be informed about sexual rehabilitation using vaginal dilators. Regarding the use of vaginal dilators, participants thought that dilators should be prescribed with the rationale that regular dilator use prevents the formation of vaginal adhesions and stenosis, and keeps the vagina accessible for penetration in the future. Vaginal dilators should be used together with lubricants. Furthermore, it was thought that the frequency of vaginal dilator use could be reduced in case the patient has resumed sexual intercourse. Health care providers, instead of the patient herself, should initiate the provision of information and patient support on this topic during follow-up appointments after treatment. Monitoring of dilator use and discussion of barriers or problems with its use should take place during each follow-up appointment.

\section{Consensus Round 2}

The panel reached consensus on 31 of 52 items in round 2 (60\%; Supplemental Digital Content 1$)$ with an equal degree of consensus between the 3 specialisms. After round 2, 14 items were formulated differently based on comments by participants. In addition, 1 item was removed from the questionnaire because this question was less specific than another item addressing the same subject.

The participants agreed that it is part of their responsibility to provide practical advice on how to cope with sexual problems. Patients treated with brachytherapy in combination with external beam RT (or on individual indications) and also patients without a current partner, especially those with cervical and vaginal cancer, should be informed about sexual rehabilitation using vaginal dilators. Patients may start having sexual intercourse 2 to 4 weeks after treatment completion. Most participants also recommended use of dilators with the rationale that dilator use makes future vaginal examinations during follow-up appointments less inconvenient. Two participants added, however, that this is not an argument to prescribe vaginal dilators, in case a patient would have no other reason to use dilators. Furthermore, participants agreed that it is not desirable to recommend use of vaginal dilators only after vaginal adhesions have been established. According to the panel, the most suitable dilators were commercially available plastic dilator sets. Vaginal dilators should be used 2 to 3 times a week during 9 to 12 months after treatment. It could help to move and rotate the dilator around when inserted. The panel agreed that gradually using a bigger cylinder circumference in time is important.

An introduction with general information about vaginal dilation should be given before the start of RT. There was consensus that the oncology nurse is the most appropriate professional to provide more extensive information and patient support during sexual rehabilitation and the use of dilators. However, in each cancer center, all gynecological oncology experts should be involved in multidisciplinary care and provide patient information, counseling, and support during sexual rehabilitation.

\section{Consensus Round 3}

Consensus was reached for 8 of 21 items in round 3 (38\%; Supplemental Digital Content 1), and the degree of consensus was equal between the 3 specialisms. The panel agreed that it is best if radiation oncologists give the first introduction and information about vaginal dilation. More extensive information should be provided during the first posttreatment follow-up appointment. According to the panel, the use of vaginal dilators can also help patients to reduce fear of bodily changes and fear of sexual activity. If preferred, patients can use a vibrator as a vaginal dilator. Furthermore, health care providers should counsel patients on which type(s) of dilator they can use, but the patient ultimately decides. To prevent adhesions, inserting the vaginal dilator for 1 to 3 minutes was thought to be sufficient. Table 2 summarizes the results and consensus-based recommendations of the study.

\section{No Consensus}

No agreement was reached on whether or not to provide standard information about sexual rehabilitation using vaginal dilators to vulvar and endometrial cancer patients, 
TABLE 2. Summary of the consensus-based recommendations described per category

Category Consensus

Responsibility

Target population

Vaginal dilator

Rationale

Content instructions

Information provision
- Health care providers should give patients simple sexological advice, ${ }^{*}$ such as how to cope with fear for sexual contact after treatment. $\dagger$

-It is desirable to refer patients to a sexologist in case simple sexological advice does not suffice. $\dagger$

Patients should be informed about vaginal dilation in case they were:

-Sexually active before treatment $\dagger$ (independent of whether they have a partner*十)

- Treated with RT for cervical $\dagger$ or vaginal cancer $\dagger$

-Treated with vaginal brachytherapy in combination with external beam RT

(or on individual indications)*

-Health care providers should advise on which type of dilator should be used, but the patient ultimately decides.

-The most often recommended type of dilator are commercially available plastic dilator sets.*

-Patients may use a vibrator if preferred.

- The circumference of a dilator is important during usage. $\dagger$

The rationale that health care providers use to prescribe vaginal dilation should contain that dilation:

-Prevents the formation of vaginal adhesions $\dagger$

-Keeps the vagina accessible for any form of penetration in the future $\dagger$

-Also makes future vaginal examination (during follow-up appointments) more convenient*

- Can be useful to help reduce fear for bodily changes and sexual activity:

-Vaginal dilation should start preventively $\dagger$ and not only in case of established adhesion.*

-Plastic cylinders, vibrators, dildos, and fingers should be inserted at least 1 to 3 minutes,

2 to 3 times a week,* and during 9 to 12 months after treatment.*

-Vaseline tampons (tampons covered in Vaseline) should be inserted overnight,*

2 to 3 times a week, ${ }^{*}$ and during at least 9 to 12 months after treatment. +

-Lubricants should be advised together with vaginal dilators. $\dagger$

-Gradually using a bigger cylinder circumference in time is important.*

-It is best to insert vaginal dilators as deep as possible, $\dagger$ in a position determined by the patient herself, $\dagger$ and to move the dilator around when inserted.*

-Patients should consult their health care provider in case of new complaints about pain $\dagger$ or lasting loss of blood. $\dagger$

-Whether or not the partner is actively involved should depend on the patients' needs. $\$$

-The frequency of use can be lowered in case the patient also has successful sexual intercourse. $\dagger$

-Patients may start having sexual intercourse 2 to 4 weeks after treatment.*

-The health care center decides which health care provider is responsible for informing patients about vaginal dilation.*

-The radiation oncologist + should provide the first introduction $\ddagger$ before RT.*

-The oncology nurse should provide the more extensive information* during the first follow-up appointment.

-The health care provider should initiate information provision, at least face-to-face, $\dagger$ even if the patient does not begin to talk about it. $\dagger$

-The patients' partners should be involved.*

-The availability of an informational brochure $\dagger$ and Web site $\dagger$ is desirable. 
TABLE 2. (Continued)

Category Consensus

Patient support
-The health care center decides which health care provider is responsible for supporting patients during sexual rehabilitation.*
-Monitoring vaginal dilator use should always take place during follow-up appointments. $\dagger$
-The oncology nurse should provide psychological and practical patient support during sexual rehabilitation.*
-The health care provider should initiate providing patient support even if the patient does not take the initiative. $\dagger$
-Extra consultations to support the patient should be possible.*
-Extra referral possibilities $\dagger$ for patients with sexual problems and more training possibilities in assessing sexual complaints* are desirable.

\footnotetext{
*Consensus reached in round 2 .

$\nmid$ Consensus reached in round 1 .

$\$$ Consensus reached in round 3 .
}

patients older than 70 years, and patients who were not sexually active before treatment (Supplemental Digital Content 2, http://links.lww.com/IGC/A231). Some participants recommended tailoring the information for these patient groups depending on the specific type(s) of treatment, age, wish to retain sexual activity, and personal and medical situation. Some participants commented to inform every patient about sexuality and vaginal dilator use after treatment with regard to possible needs in the future. Furthermore, participants did not agree on whether dilation should be started between 2 and 4 weeks after RT $(n=20 ; 67 \%)$ or later. It was commented that it is important for the vaginal mucosa to have healed before dilation is started, which often takes 4 weeks after completion of RT.

\section{DISCUSSION}

A 3-round online Delphi study was conducted to assess the content and procedures of patient information provision and support regarding sexual rehabilitation and vaginal dilator use after RT for gynecological cancer. The study was conducted among 30 gynecologic oncology experts from 12 gynecological cancer centers. The panel equally represented radiation oncologists, gynecologic oncologists, and specialized oncology nurses involved and experienced in counseling and the follow-up of gynecological cancer patients after RT. All participants completed the 3 rounds. The degree to which consensus was reached was equal between the 3 professional groups. Previously, no specific recommendations could be made regarding the content and procedures of information provision and support during sexual rehabilitation and vaginal dilator use. ${ }^{16,22,23}$ This study offers a clear consensus on these topics.

Consensus was reached that information about sexual rehabilitation and vaginal dilator use should be given to all sexually active cervical and vaginal cancer patients younger than 70 years. Moreover, comments by the participants made clear that vulvar and endometrial cancer patients, patients older than 70 years, and patients who were not sexually active before treatment should receive care tailored to their needs. The participants also agreed that radiation oncologists should initiate and provide information about sexual adverse effects of RT, rehabilitation, and preventive measures including vaginal dilator use before treatment. In addition, in line with previous suggestions, ${ }^{30}$ more extensive dedicated psychological and practical support should preferably be initiated and given by specifically trained oncology nurses.

Although in this study, no clear consensus was reached on the best time to start dilator use, most participants recommended to start between 2 and 4 weeks after completion of RT $(\mathrm{n}=20 ; 67 \%)$ or as soon as the vaginal mucosa is healed (which is usually around 4 weeks). This was also the most commonly cited time interval in the studies of White and Faithfull $^{22}$ and Lancaster. ${ }^{23}$ Furthermore, there was consensus that the frequency with which dilators should be used, preferably 2 to 3 times a week, could be reduced in case the patient has resumed sexual intercourse.

The panel agreed that it is important to gradually use a bigger cylinder circumference in time. Participants in this study also agreed that each dilator should be inserted during 1 to 3 minutes, which is in contrast to previous recommendations of 5 to 10 minutes. ${ }^{16,22,23}$ Furthermore, the panel agreed that it is best to perform dilation for 9 to 12 months, whereas in previous studies, about half of the respondents recommended indefinite use. ${ }^{22,23}$ However, in contrast to the aforementioned survey studies, ${ }^{22,23}$ this present study conducted the Delphi method among a heterogeneous panel. Therefore, the results of this study were thought to reflect the opinion of all professionals involved in counseling and the follow-up of gynecological cancer patients after RT.

Adapting items for the next round in the Delphi study obviously poses a risk of a self-fulfilling prophecy. ${ }^{25}$ However, the items concerned were adapted using experts' comments in a systematic way, and all the adapted items were pilot-tested on comprehensibility again among 5 experienced researchers in the field. The professionals that participated in this study were expected to be potential users of the findings and, consequently, were thought to form the most useful 
expert panel. In a separate in-depth interview study, the perspective of gynecological cancer patients on sexual rehabilitation and dilator use after pelvic RT will be investigated. The patient's perspective on this topic is required to be able to improve patient care because it is expected that these patients have additional needs. For example, participants in this study considered the rigid plastic dilator sets as the most suitable type of dilator to prescribe, whereas patients might have preferred a softer flexible dilator. ${ }^{21}$ The efficacy of regular dilator use can only be assessed in a randomized controlled trial if there is a standardized procedure of sexual rehabilitation and vaginal dilator use. Using the Delphi method, a common consensus was created out of the available expertise. It is therefore important to further investigate whether the results of this study are generally applicable in clinical practice and support patients during their sexual rehabilitation after pelvic RT before final policy decisions can be made.

In conclusion, the results of this study offer clear consensus-based recommendations for the education and support of gynecological cancer patients treated with pelvic RT during sexual rehabilitation and vaginal dilator use. Based on these results, an intervention has been developed and is currently pilot-tested in which patients receive support during sexual rehabilitation and vaginal dilator use after pelvic RT. This study was a first step toward the improvement of gynecological cancer patient support during their sexual rehabilitation after pelvic RT.

\section{ACKNOWLEDGMENTS}

The authors would like to thank all participating radiation oncologists, gynecologic oncologists, and oncology nurses for their effort and time investment. The authors would also like to thank Professor Dr A.M. Stiggelbout (Department of Medical Decision Making, Leiden University Medical Center, the Netherlands) for helping us pilot-test the items in the questionnaire used in this study.

\section{REFERENCES}

1. Vereniging van Integrale Kankercentra. Dutch Cancer Registry, 2011. Available at: http://www.cijfersoverkanker.nl. Accessed December 24, 2013.

2. Waggoner SE. Cervical cancer. Lancet. 2003;361:2217-2225.

3. Abbott-Anderson K, Kwekkeboom KL. A systematic review of sexual concerns reported by gynecological cancer survivors. Gynecol Oncol. 2012;124:477-489.

4. Lindau ST, Gavrilova N, Anderson D. Sexual morbidity in very long term survivors of vaginal and cervical cancer: a comparison to national norms. Gynecol Oncol. 2007;106: 413-418.

5. Pieterse QD, Maas CP, ter Kuile MM, et al. An observational longitudinal study to evaluate miction, defecation, and sexual function after radical hysterectomy with pelvic lymphadenectomy for early-stage cervical cancer. Int J Gynecol Cancer. 2006;16:1119-1129.

6. Jensen PT, Groenvold M, Klee MC, et al. Early-stage cervical carcinoma, radical hysterectomy, and sexual function-a longitudinal study. Cancer. 2004;100:97-106.
7. Bergmark K, Avall-Lundqvist E, Dickman PW, et al. Vaginal changes and sexuality in women with a history of cervical cancer. N Engl J Med. 1999;340:1383-1389.

8. Pieterse QD, Kenter GG, Maas CP, et al. Self-reported sexual, bowel and bladder function in cervical cancer patients following different treatment modalities: longitudinal prospective cohort study. Int J Gynecol Cancer. 2013;23:1717-1725.

9. Frumovitz M, Sun CC, Schover LR, et al. Quality of life and sexual functioning in cervical cancer survivors. J Clin Oncol. 2005;23:7428-7436.

10. Jensen PT, Groenvold M, Klee MC, et al. Longitudinal study of sexual function and vaginal changes after radiotherapy for cervical cancer. Int J Radiat Oncol Biol Phys. 2003;56:937-949.

11. Brand AH, Bull CA, Cakir B. Vaginal stenosis in patients treated with radiotherapy for carcinoma of the cervix. Int J Gynecol Cancer. 2006;16:288-293.

12. Gondi V, Bentzen SM, Sklenar KL, et al. Severe late toxicities following concomitant chemoradiotherapy compared to radiotherapy alone in cervical cancer: an inter-era analysis. Int J Radiat Oncol Biol Phys. 2012;84:973-982.

13. Velaskar SM, Martha R, Mahantashetty U, et al. Use of indigenous vaginal dilator in radiation induced vaginal stenosis. Indian J Occup Ther. 2007;39:3-6.

14. Decruze SB, Guthrie D, Magnani R. Prevention of vaginal stenosis in patients following vaginal brachytherapy. Clin Oncol. 1999;11:46-48.

15. Miles T, Johnson N. Vaginal dilator therapy for women receiving pelvic radiotherapy. Cochrane Database Syst Rev. 2010;9:1-29.

16. Clinical Guideline Group. International Guidelines on Vaginal Dilation After Pelvic Radiotherapy 2012. Oxon, Owen Mumford. Available at: http://www.ncsi.org.uk/wp-content/ uploads/Inter-Best-Practice-Guide-Vaginal-Dilators-July-2012. pdf. Accessed February 12, 2014.

17. Grigsby PW, Russell A, Bruner D, et al. Late injury of cancer therapy on the female reproductive tract. Int $J$ Radiat Oncol Biol Phys. 1995;31:1281-1299.

18. Crowther ME, Corney RH, Shepherd JH. Psychosexual implications of gynecological cancer. BMJ. 1994;308: 869-870.

19. Cullen K, Fergus K, Dasgupta T, et al. Toward clinical care guidelines for supporting rehabilitative vaginal dilator use with women recovering from cervical cancer. Support Care Cancer. 2013;21:1911-1917.

20. Bonner C, Nattress K, Anderson C, et al. Chore or priority? Barriers and facilitators affecting dilator use after pelvic radiotherapy for gynaecological cancer. Support Care Cancer. 2012;20:2305-2313.

21. Juraskova I, Butow P, Robertson R, et al. Post-treatment sexual adjustment following cervical and endometrial cancer: a qualitative insight. Psychooncology. 2003;12: 267-279.

22. White ID, Faithfull S. Vaginal dilation associated with pelvic radiotherapy: a UK survey of current practice. Int J Gynecol Cancer. 2006;16:1140-1146.

23. Lancaster L. Preventing vaginal stenosis after brachtherapy for gynaecological cancer: an overview of Australian practices. Eur J Oncol Nurs. 2004;8:30-39.

24. Keeney S, Hasson F, McKenna H. Consulting the oracle: ten lessons from using the Delphi technique in nursing research. J Adv Nurs. 2006;53:205-212. 
25. Greatorex J, Dexter T. An accessible analytical approach for investigating what happens between the rounds of a Delphi study. J Adv Nurs. 2000;32:1016-1024.

26. Murphy MK, Black NA, Lamping DL, et al. Consensus development methods, and their use in clinical guideline development. Health Technol Assess. 1998;2:i-iv, 1-88.

27. Jones J, Hunter D. Consensus methods for medical and health services research. BMJ. 1995;311:376-380.
28. Linstone HA, Turoff M. The Delphi Method Techniques and Applications. Reading, MA: Addison Wesley; 1975.

29. Faithfull S, White I. Delivering sensitive health care information: challenging the taboo of women's sexual health after pelvic radiotherapy. Patient Educ Couns. 2008;71:228-233.

30. Booth K, Beaver K, Kitchener H, et al. Women's experiences of information, psychological distress and worry after treatment for gynaecological cancer. Patient Educ Couns. 2005;56:225-232. 